\title{
QUIT: QUantitative Imaging Tools
}

DOI: $10.21105 /$ joss.00656

\section{Software}

- Review ¿

- Repository ¿

- Archive ct

Submitted: 27 March 2018

Published: 21 June 2018

\section{License}

Authors of papers retain copyright and release the work under a Creative Commons Attribution 4.0 International License (CC-BY).

\section{Tobias C Wood $^{1}$}

1 Department of Neuroimaging, King's College London

\section{Summary}

- Magnetic Resonance Imaging (MRI) is evolving from acquiring simple qualitative images towards quantitative data, where information can be inferred about tissue microstructure within each voxel individually.

- Many papers written in this area do not make processing code available. This hampers adoption of valuable quantitative techniques outside the groups where they are developed. There is hence a need for open-source implementations of common quantitative methods.

- QUIT (http://github.com/spinicist/QUIT) provides implementations of multiple quantitative MRI algorithms as command-line tools written in $\mathrm{C}++$ and using the high-quality Insight Toolkit (Johnson, Hans J 2015), CERES (Agarwal, Sameer, n.d.) and Eigen (Guennebaud, Gaël 2010) libraries. They are multi-threaded whereever possible. They are easy to include into shell-script based processing pipelines, and are easy to use with a queue system such as the Sun Grid Engine. They are hence suitable for the high-throughput processing that is required for timely analysis of a large neuroimaging study.

- The recent qMRLab project (https://github.com/neuropoly/qMRLab) has similar aims and provides a large variety of techniques written in Matlab. Although Matlab is widespread in academic environments, it is not always available. In addition, the primary focus of qMRLab is educational while the focus for QUIT is highthroughput processing of multiple datasets.

- QUIT is currently in use for several ongoing studies at multiple sites and has been used during the preparation of several manuscripts(Wood, Tobias C. Simmons, Camilla 2016)(Hawkins, Peter C.T. 2017)(Richetto, Juliet 2017)(Vanes, Lucy D 2018).

\section{References}

Agarwal, Sameer, Others, Mierle, Keir. n.d. "Ceres Solver." http://ceres-solver.org. Guennebaud, Gaël, Jacob, Benoît. 2010. "Eigen V3.” http://eigen.tuxfamily.org.

Hawkins, Peter C.T., Vernon, Anthony C., Wood, Tobias C. 2017. "An Investigation of Regional Cerebral Blood Flow and Tissue Structure Changes After Acute Administration of Antipsychotics in Healthy Male Volunteers." http://doi.wiley.com/10.1002/hbm.23844. https://doi.org/10.1002/hbm.23844.

Johnson, Hans J, Ibanez, Luis, McCormick, Matthew M. 2015. Template:The Itk Software Guide Book 1: Introduction and Development Guidelines-Volume 1. Kitware, Inc.

Richetto, Juliet, Cattaneo, Annamaria, Chesters, Robert. 2017. "Genome-Wide Transcriptional Profiling and Structural Magnetic Resonance Imaging in the Maternal Immune 
Activation Model of Neurodevelopmental Disorders." https://doi.org/10.1093/cercor/ bhw320.

Vanes, Lucy D, Wood, Tobias C, Mouchlianitis, Elias. 2018. "White Matter Changes in Treatment Refractory Schizophrenia: Does Cognitive Control and Myelination Matter?" https://doi.org/10.1016/j.nicl.2018.01.010.

Wood, Tobias C. Simmons, Camilla, Vernon, Anthony C., Hurley, Samuel A. 2016. "Whole-Brain Ex-Vivo Quantitative Mri of the Cuprizone Mouse Model." https://doi. org/10.7717/peerj.2632. https://doi.org/10.7717/peerj.2632. 